\title{
Intracardiac Metastasis from a Large Cell Neuroendocrine Lung Carcinoma
}

\author{
Christopher Gaisendrees $^{1 \oplus}$ Kaveh Eghbalzadeh $^{1}$ Navid Mader $^{1} \quad$ Thorsten C. W. Wahlers $^{1}$ \\ ${ }^{1}$ Herz- und Thoraxchirurgie, Uniklinik Koln, Koln, Germany \\ Address for correspondence Christopher Gaisendrees, Herz- und \\ Thorac Cardiovasc Surg Rep 2019;8:e41-e43. \begin{abstract}
Thoraxchirurgie, Uniklinik Koln, Kerpener Street 62, Koln 50937,
\end{abstract} \\ Germany (e-mail: Christopher.gaisendrees@uk-koeln.de).
}
Abstract
Keywords
- cardiac tumor
- cardiovascular
- surgery
- metastases
- lung cancer
- heart
- tricuspid valve

Primary malignant tumors of the heart are rare; the biggest group is sarcomas. Cardiac metastases make up the biggest group of secondary cardiac tumors. We present a rare case of cardiac metastasis $(3.1 \times 3.2 \times 2.8 \mathrm{~cm})$ localized in the right atrium, originating from a large cell neuroendocrine lung carcinoma, with close contact to the tricuspid valve and inferior cava vein.

\section{Introduction}

Primary tumors of the heart are rare, with an incidence of around $0.2 \%$ in unselected patients at autopsy. ${ }^{1-3}$ Even though the occurrence of cardiac tumors is well described in medical literature, diagnosing it remains challenging in clinical practice. The majority (75\%) of these tumors are benign; nearly half of those are myxomas. Cardiac sarcomas are the biggest group of primary malignant cardiac tumors and cardiac metastases (CMs) make up the biggest group in secondary malignant cardiac tumors. They can be observed in 10 to $20 \%$ of patients suffering from a disseminated malignant tumor disease. ${ }^{4}$

Among pathology of malignant lung cancer, large cell neuroendocrine lung carcinoma (LCNEC) is very rare with a reported range of 2.4 to $3.1 \%$ in resected lung cancers. ${ }^{5,6}$ More than $90 \%$ of $\mathrm{CM}$ are clinically silent, thus they are rarely detected before death. ${ }^{7}$

\section{Case Description}

A 53-year-old patient was transferred to our department for workup of an unknown cardiac mass located close to the heart. The suspected mass was accidentally diagnosed in positron emission tomography (PET)-computed tomography (CT) scan. The examination was initially performed to scan for metastasis of a suspected malignant tumor of the right superior pulmo- nary lobe, which was diagnosed earlier that year. PET-CT scan showed nuclide accumulation in the right superior lobe, the left suprarenal gland, and below the heart on the top of the pericardium. The magnetic resonance imaging (MRI) of the neurocranium showed no pathological abnormalities.

Following the diagnosis, an explorative thoracotomy with lobectomy of the right superior lobe and resection of the right dorsobasal inferior lobe was performed. Acquired intraoperative biopsy showed LCNEC (70\%) with components of adenocarcinoma (30\%) and involved visceral pleura. TNM classification of the tumor was rated as pT2b, pNO (0/15), L0, V0, R0, G3. After surgical removal of the primary tumor in the lung, excision of the metastasis located in the left suprarenal gland was performed.

After treatment of the lung and suprarenal gland, cardioMRI scan was performed to further classify the suspected mass which was initially observed in the former PET-CT scan. MRI scan revealed a suspected tumor mass of $3.1 \times 3.2 \times 2.8$ $\mathrm{cm}$, borderline close to the inferior cava vein and the tricuspid valve (-Fig. 1 )

Additionally, the transesophageal echocardiography (TEE) showed a tumor in the right atrium ( - Figs. 2 and $\mathbf{3}$ ), originating from the lateral wall reaching the border of the tricuspid valve.

At admission for surgery, the patient showed not to be frail (World Health Organization I) at normal nutritional
License terms Stuttgart · New York

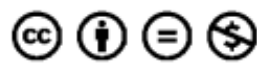

received

July 5, 2019

accepted

August 9, 2019
DOI https://doi.org/

10.1055/s-0039-1698406. ISSN 2194-7635. 


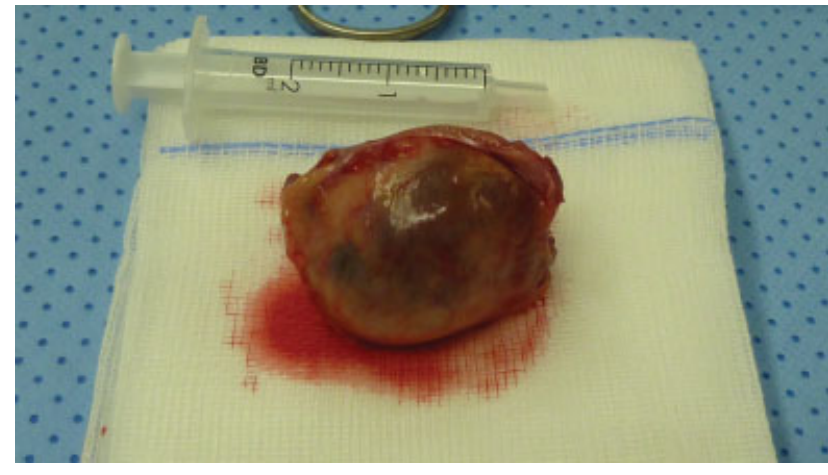

Fig. 1 Resected metastasis.

status ( $193 \mathrm{~cm}$ and $92 \mathrm{~kg}$ ). No pathological heart sounds were present at auscultation. The patient reported nausea and intermitting dyspnea after formerly performed bilobectomy. Arterial hypertension was found in the patient's medical history. The chest X-ray showed no signs of enlarged heart silhouette. Electrocardiogram (ECG) showed no signs of arrhythmia.

In consequence, operative tumor resection was done using full median sternotomy. Venous cannulation for the heart-lung machine was achieved by cannulation of right femoral for the lower drainage, and additionally, the superior caval vein was cannulated to ensure full exposure of the right atrium with arterial cannulation of the ascending aorta as well. After full heparinization and cross-clamping, cardioplegic arrest was induced by using Buckberg's solution. After opening the right atrium, a large tumor, located at the inferior cava vein, with contact to the tricuspid valve (-Fig. 4) was exposed. Additionally, the tumor was shown to be located in direct proximity to the descending part of the right coronary artery onto the border of the coronary sinus. The tumor was completely extirpated along the right coronary artery and the tricuspid valve. Due to the fact that the tumor prolapsed into the inferior leaflet of the tricuspid valve, a 34-mm Edwards MC3 ring was implanted to stabilize the valve at its base. Furthermore, a pericardial patch of $12 \times 5 \mathrm{~cm}$ was used to cover the defect of the right atrium, sewing it partially to the MC3 ring by using 4.0 Prolene. Then,

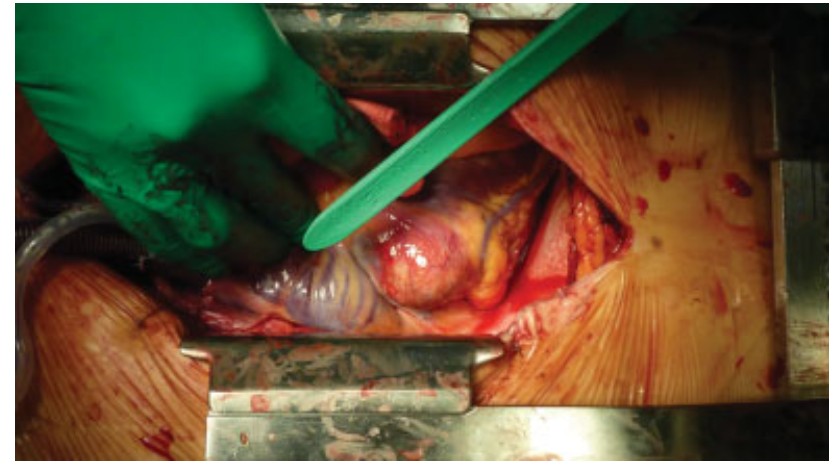

Fig. 2 Intraoperative view.

the heart was antegradely deaired. The unknown tumor mass was sent to the pathology laboratory for further examination. Bypass time was 111 minutes with a crossclamp time of 84 minutes and a usage of $900 \mathrm{~mL}$ Buckberg. Molecular, biological, and pathological reports verified the occurrence of metastasis from an LCNEC. In the postoperative course, TTE showed a low gradient over the implanted tricuspid valve ( $P$ (pressure) mean $2.5 \mathrm{~mm} \mathrm{Hg}$ ) and a good left ventricular ejection fraction. The patient recovered well from surgery and further treatment of the primary lung tumor was initiated by using chemotherapy 1 week after surgery.

\section{Discussion}

Malignant lung cancer is one of the most common types of cancer and the most common cause of cancer-related mortality. Metastasis from lung cancer spread via lymphatic and hemorrhagic distribution. The hematological spreading of metastasis occurs in the right atrium through caval veins and in the left atrium by pulmonary veins, although much less common.

The rate of metastasation from lung cancer to the heart varies with the histology of the primary tumor and is described with a probability of $\sim 20 \%{ }^{7}$

Neuroendocrine neoplasia of the lung is extremely rare and makes up 1 to $2 \%$ of all lung tumors and 20 to $30 \%$ of neuroendocrine tumors. $^{8}$ Most affected areas of lung

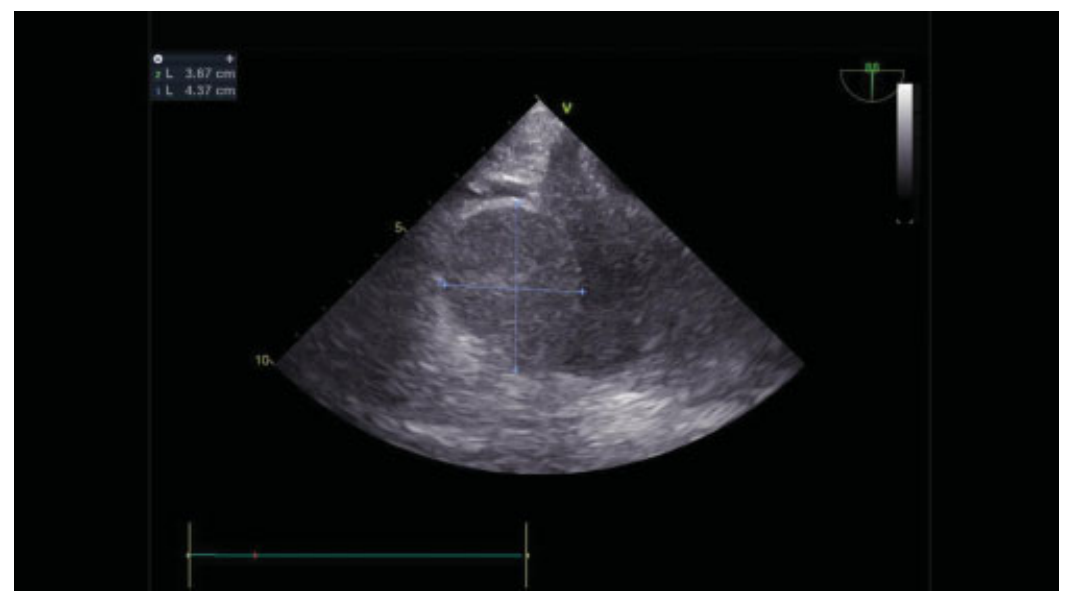

Fig. 3 TEE showing large intracardiac mass of $3.67 \times 4.37 \mathrm{~cm}$. 


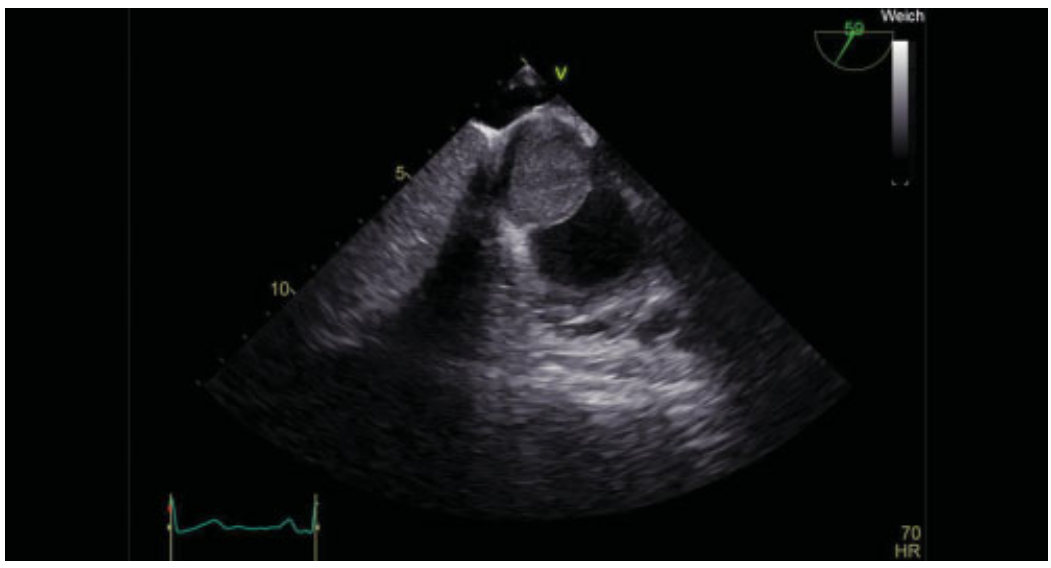

Fig. 4 TEE showing large intracardiac metastasis.

metastases include liver, brain, bone, lymph nodes, and adrenal glands.

The possibility of CM should be an important consideration in patients with malignancies and newly observed cardiovascular-related symptoms. Although clinically mostly silent, physical examination may guide the way to diagnosis. A newly occurred heart sound, for example, may suggest a potential intracardiac mass. ECG should be a standard examination tool, although nonspecific, ST-wave changes, and new arrhythmias can lead the way to further examination.

Imaging is essential for the diagnosis of CM. Conventional chest X-ray is mostly unspecific but might show cardiomegaly.

Echocardiography is the imaging of choice; it provides the most accurate information on the exact localization and anatomy of the cardiac tumor. Nevertheless, besides the possibility of a tumor, the most likely diagnosis of an intracardiac mass is a thrombus or vegetation.

Considering the treatment, surgical resection is generally performed in cases with an otherwise good prognosis and in cases where surgical resection is technically feasible and safe. Resection through minimally invasive approaches is also possible, depending on the size and location of the tumor.
Conflict of Interest

None.

\section{References}

1 Lam KY, Dickens P, Chan AC. Tumors of the heart. A 20-year experience with a review of 12,485 consecutive autopsies. Arch Pathol Lab Med 1993;117(10):1027-1031

2 Buja LM. The multifaceted manifestations of cardiac tumors. Tex Heart Inst J 2012;39(01):84-85

3 Wenger NK. Pericardial disease in the elderly. Cardiovasc Clin 1992;22(02):97-103

4 McAllister HA, Fenoglio JJ. Tumors of the Cardiovascular System. Atlas of Tumor Pathology, Series 2. Washington DC: Armed Forces Institute of Pathology; 1978

5 Iyoda A, Hiroshima K, Toyozaki T, Haga Y, Fujisawa T, Ohwada H. Clinical characterization of pulmonary large cell neuroendocrine carcinoma and large cell carcinoma with neuroendocrine morphology. Cancer 2001;91(11):1992-2000

6 Takei H, Asamura H, Maeshima A, et al. Large cell neuroendocrine carcinoma of the lung: a clinicopathologic study of eighty-seven cases. J Thorac Cardiovasc Surg 2002;124(02):285-292

7 Bussani R, De-Giorgio F, Abbate A, Silvestri F. Cardiac metastases. J Clin Pathol 2007;60(01):27-34

8 Quaedvlieg PF, Visser O, Lamers CB, Janssen-Heijen ML, Taal BG. Epidemiology and survival in patients with carcinoid disease in The Netherlands. An epidemiological study with 2391 patients. Ann Oncol 2001;12(09):1295-1300 